\title{
Diagrammatic expansion of information flows in stochastic Boolean networks
}

\author{
Fumito Mori॰* \\ Faculty of Design, Kyushu University, Fukuoka 815-8540, Japan \\ and Education and Research Center for Mathematical and Data Science, Kyushu University, Fukuoka 819-0395, Japan \\ Takashi Okada $\odot^{\dagger}$ \\ RIKEN Interdisciplinary Theoretical and Mathematical Sciences Program (iTHEMS), Wako 351-0198, Japan \\ and Department of Physics and Department of Integrative Biology, University of California, Berkeley, California 94720, USA
}

(Received 30 June 2020; revised 21 July 2020; accepted 10 December 2020; published 29 December 2020)

\begin{abstract}
Accurate information transfer is essential for biological, social, and technological networks. Computing transfer entropy (TE), a measure of information flow, usually relies on numerical methods even in small networks, which obscures the origin of accurate information transfer. In this study, we establish a diagrammatic formula that analytically computes TE for a stochastic Boolean network, where the intermediate network from signal to output can have arbitrary topology and nonidentical Boolean functions. By expressing TE in terms of network components, we elucidate the mechanism of information flow and provide optimal design principles of network architectures applicable to real networks.
\end{abstract}

DOI: 10.1103/PhysRevResearch.2.043432

\section{INTRODUCTION}

In biological systems, signals from the environment are transmitted over large complex networks. Examples include gene regulatory networks, signal transduction networks, and neural networks. They are modeled using Boolean dynamics when expressions in terms of binary states are sufficient to extract their characteristics [1-12]. Biological functions rely on how accurately subsystems in the networks can communicate with each other. Biological networks often possess characteristic topology, such as small-world and scale-free properties [13,14] and motifs [15-18]. They also have regulatory functions that are highly biased, in favor of either high or low frequency in on-state outputs $[19,20]$, and high frequency of canalizing inputs [21,22]. Another well-studied class of functions is monotonic functions [23,24]. These characteristic features are thought to allow accurate communications.

Transfer entropy (TE) is an information-theoretic measure of how the process of one subsystem depends on others, originally introduced in [25]. Unlike simple correlations between variables, TE can detect causal influence between components and is suitable to quantify the accuracy of signal transfer. It is studied in nonequilibrium thermodynamics [26-29]. It is applied in many other scientific contexts, such as chemical reactions [29-31], neuroscience [32,33], economics [34-36],

\footnotetext{
*mori@design.kyushu-u.ac.jp

†takashi.okada@riken.jp
}

Published by the American Physical Society under the terms of the Creative Commons Attribution 4.0 International license. Further distribution of this work must maintain attribution to the author(s) and the published article's title, journal citation, and DOI. and machine learning [37]. There have been attempts to extend the TE as a more robust measure of information transfer [38-40].

While TE is a commonly used measure of information flow, the origins of accurate information transfer remain obscure. Most theoretical studies use methods that, in principle, generalize to a wide class of networks, but are demonstrated only in small networks (or low-dimensional phenomenological systems when the actual systems are highly complicated, e.g., $[27-29,31,41])$. One of the reasons for this is that analytical computation of TE involves high-dimensional matrix computation. As an illustration, consider the so-called coherent and incoherent motifs shown in Fig. 1(a). They often appear in gene regulatory networks (see, e.g., [42]). If each variable (gene), represented by a vertex, takes two states, $\mathrm{ON}$ or OFF, the whole system has $2^{4}$ dimensions. More generally, when there are $N$ variables, the size becomes $2^{N}$-dimensional. Thus, analytical computation of TE quickly becomes infeasible as the system size increases. Another drawback of the matrix computation is that it does not provide any physical insights into the mechanism of information flow. Figure 1(b) shows TE, obtained numerically, for the two motifs in Fig. 1(a). However, the matrix computation does not answer why the TE of the coherent motif is higher than that of the incoherent motif. There have been several attempts to calculate TE analytically in large complex network elements [43-45]. However, in these studies, it was assumed that elements are identical and obey simple dynamics. A systematic formula of TE for general network dynamics is still lacking.

In this paper, we establish an analytical method that allows the computation of TE for a stochastic Boolean network without the need for performing matrix algebra, and is applicable to a general Boolean network, where the intermediate network connecting the signal source and output can have 
(a)

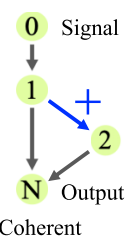

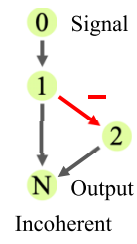

(b)

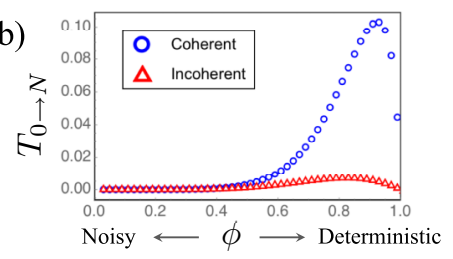

(c) $T_{0 \rightarrow N}=\frac{1}{2} \mathcal{P}_{3}^{2}+\frac{1}{4 \cdot 3}\left(\begin{array}{l}4 \\ 2\end{array}\right) \mathcal{P}_{1}^{2} \mathcal{P}_{3}^{2}+\frac{1}{2}\left(\begin{array}{l}2 \\ 1\end{array}\right) \mathcal{P}_{3} \mathcal{P}_{4}+\mathcal{O}\left(\phi^{10}\right)$

$$
=\mathcal{P}_{3}^{2}\left\|+\mathcal{P}_{3}^{2}\right\|_{\mathcal{P}_{1}^{2}}+\mathcal{P}_{3} \|_{\mathcal{P}_{4}}+\cdots
$$

FIG. 1. (a) A coherent motif with Boolean functions $f_{1}\left(x_{0}\right)=x_{0}$, $f_{2}\left(x_{1}\right)=x_{1}$, and $f_{N}\left(x_{1}, x_{2}\right)=\left(x_{1}\right.$ AND $\left.x_{2}\right)$ and an incoherent motif identical except that $f_{2}\left(x_{1}\right)=\overline{x_{1}}$. The coherent motif has positive interactions both for the direct and indirect routes from the signal source to the output vertex, whereas the incoherent motif has positive and negative interactions for the direct and indirect routes, respectively. Transfer entropy (TE) from signal source labeled 0 to output vertex labeled $N$ is considered. (b) Numerically obtained $T_{0 \rightarrow N}$ as a function of $\phi$ in the two motifs. (c) Schematics of diagrammatic expansion formula of $T_{0 \rightarrow N}$, using pathway weight $\mathcal{P}_{\alpha}$ (see Fig. 2). The crosstalk between $\mathcal{P}_{3}$ and $\mathcal{P}_{4}$ yields a difference in TE between the two motifs (a).

arbitrary topology and an arbitrary set of Boolean functions. An important feature of our method is that it performs a diagrammatic expansion by expressing TE in terms of many possible graphical structures (or pathways, defined later) and then eliminating most structures based on certain graphical conditions. Consequently, the relevant pathway combinations for the information flow are extracted. In other words, our method elucidates how the information flow is produced via crosstalk between pathways [see schematic in Fig. 1(c)]. Furthermore, based on our analytical results, we provide the optimal design principles of network architectures applicable to real complex networks.

\section{MODEL DEFINITION}

We consider a stochastic Boolean network with discrete time described as

$$
x_{i}^{t+1}= \begin{cases}f_{i}\left(\boldsymbol{x}_{\boldsymbol{I}_{i}}^{t}\right) & \text { with probability } \frac{1}{2}\left(1+\phi_{i}\right), \\ f_{i}\left(\boldsymbol{x}_{\boldsymbol{I}_{i}}^{t}\right) & \text { with probability } \frac{1}{2}\left(1-\phi_{i}\right),\end{cases}
$$

where $x_{i}^{t}$ is a Boolean variable of vertex $i$ at a time $t$, and $f_{i}$ is a Boolean function. The negation of a Boolean variable $x$ is denoted by $\bar{x}$. A set of input vertices to vertex $i$ is denoted as $\boldsymbol{I}_{i}$, and a set of Boolean variables of $\boldsymbol{I}_{i}$ at $t$ is denoted as $\boldsymbol{x}_{\boldsymbol{I}_{i}}^{t}$. We assign 0 and $N$ to the signal and output vertices, respectively, and $1, \ldots,(N-1)$ to the other vertices arbitrarily [Fig. 1(a)]. Thus, a state of the whole system at $t$ is represented by $\boldsymbol{x}^{t}=\left(x_{0}^{t}, x_{1}^{t}, \ldots, x_{N}^{t}\right)$. We assume that there is no direct link from 0 to $N$. Generally, links can form cycles, and vertices except the output vertex can have self-loops. $\phi_{i}\left(0 \leqslant \phi_{i}<1\right)$ is a noise parameter of vertex $i$, i.e., the dynamics of vertex $i$ becomes deterministic in the limit of $\phi_{i} \rightarrow 1$, while it is completely random when $\phi_{i}=0$. The signal source has

the identity function $f_{0}\left(x_{0}^{t}\right)=x_{0}^{t}$, and the switching between $x_{0}=0$ and $x_{0}=1$ becomes slow in the limit of $\phi_{0} \rightarrow 1$ while fast when $\phi_{0}=0$. The vertices $i=1, \ldots, N$ can be assigned arbitrary Boolean functions. All variables are synchronously updated according to Eq. (1) at every $t$.

We explain here the reasons that the several assumptions for network links are introduced. First, it is assumed that $x_{0}$ is dependent only on itself, $f_{0}\left(x_{0}^{t}\right)=x_{0}^{t}$, because we focus on the signaling process from an environmental signal (e.g., ligands in signal transduction). Equation (1) then implies that the signal is unbiased, $P\left(x_{0}^{t}=1\right)=P\left(x_{0}^{t}=0\right)=\frac{1}{2}$. We will discuss an extension to a biased signal in Sec. VI. We note that our formulation holds even when the signal source takes inputs from other vertices. Second, we prohibit a direct link from the signal source to the output vertex, because, otherwise, the information would be transferred primarily through the direct link and the entire network structure would contribute little to TE (at least when $\phi$ is small). Third, we assume for simplicity that the output vertex does not have a self-loop. If a self-loop is allowed for the output vertex, the analysis becomes more involved, and, especially, calculation of Eq. (6) becomes complicated.

We remark on functional constraints imposed by network topology. A Boolean function $f_{i}$ should satisfy the dependency on its input variables $\boldsymbol{x}_{\boldsymbol{I}_{i}}$, i.e., for any $j \in \boldsymbol{I}_{i}$, $f_{i}\left(x_{j}=0, \boldsymbol{x}_{\boldsymbol{I}_{i} \backslash j}\right) \neq f_{i}\left(x_{j}=1, \boldsymbol{x}_{\boldsymbol{I}_{i} \backslash j}\right)$ holds for at least one state of $\boldsymbol{x}_{\boldsymbol{I}_{i} \backslash j}$ because network topology in our system Eq. (1) is

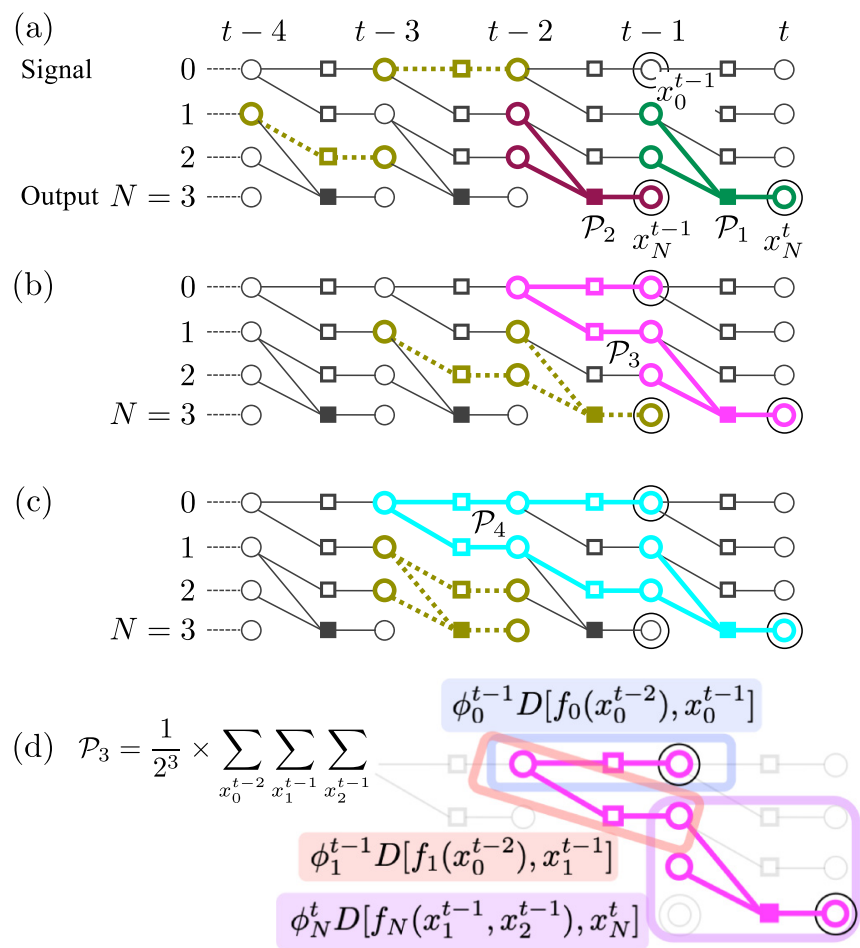

FIG. 2. Bonds and pathways in temporal graphs corresponding to the networks in Fig. 1(a), where double circles represent the external nodes $x_{N}^{t}, x_{N}^{t-1}$, and $x_{0}^{t-1}$. (a) Bonds (bold and dotted lines). Each $\mathcal{P}_{1}$ and $\mathcal{P}_{2}$ is an existent pathway comprising a single bond. (b),(c) Existent pathways $\mathcal{P}_{3}$ and $\mathcal{P}_{4}$ (bold lines) and nonexistent pathways (dotted lines). (d) Pathway weight $\mathcal{P}_{3}$, where a factor $\phi D$ is assigned to each bond, enclosed by a rectangle. 
identified by regulation relations, i.e., a set of $\boldsymbol{x}_{\boldsymbol{I}_{i}}$. This dependency condition is, however, not necessarily required for our formulation.

Entropy and conditional entropy are respectively defined as $H[\boldsymbol{x}]=-\sum_{x} P(\boldsymbol{x}) \ln P(\boldsymbol{x})$ and $H[\boldsymbol{x} \mid \boldsymbol{y}]=H[\boldsymbol{x}, \boldsymbol{y}]-H[\boldsymbol{y}]$, where $P(\boldsymbol{x})$ is a probability distribution of a set of variables $\boldsymbol{x}$. We assume a stationary signal $P\left(x_{0}^{t}\right)=P\left(x_{0}^{t^{\prime}}\right)$, and focus on the TE from $x_{0}$ to $x_{N}$ in the stationary state defined as

$$
T_{0 \rightarrow N}=H\left[x_{N}^{t} \mid x_{N}^{t-1}\right]-H\left[x_{N}^{t} \mid x_{N}^{t-1}, x_{0}^{t-1}\right],
$$

where the lag time $\Delta t=1$ is employed in the definition of TE. Based on the definition, $T_{0 \rightarrow N}$ is determined by the joint distribution $P\left(x_{N}^{t}, x_{N}^{t-1}, x_{0}^{t-1}\right)$. Although it is generally difficult to obtain the exact joint distribution, our method can estimate $T_{0 \rightarrow N}$ with arbitrary precision in a wide range of parameters $\phi_{i}$.

\section{DIAGRAMMATIC EXPANSION}

We begin by expressing the conditional probability $P\left(x_{i}^{t} \mid \boldsymbol{x}_{\boldsymbol{I}_{i}}^{t-1}\right)$ as follows:

$$
P\left(x_{i}^{t} \mid \boldsymbol{x}_{\boldsymbol{I}_{i}}^{t-1}\right)=\frac{1}{2}\left(1+\phi_{i}^{t} D\left[f_{i}\left(\boldsymbol{x}_{\boldsymbol{I}_{i}}^{t-1}\right), x_{i}^{t}\right]\right) .
$$

Here, $D[x, y] \equiv \delta[x, y]-\delta[y, \bar{x}]$, where $\delta[x, y]$ is the Kronecker delta; $\delta[x, x]=1$ and $\delta[x, \bar{x}]=0$. Note that in Eq. (3), we appended $t$ to $\phi_{i}$ as a marker to assist in our formulation.

The factor $\phi_{i}^{t} D\left[f_{i}\left(\boldsymbol{x}_{\boldsymbol{I}_{i}}^{t-1}\right), x_{i}^{t}\right]$ in Eq. (3) can be expressed graphically in a bipartite network called a temporal graph.
A similar graph was used by [26,46-48]. It is illustrated in Figs. 2(a)-2(c) for the regulatory network in Fig. 1(a). In a temporal graph, a variable $x_{i}^{t}$ is represented by a circle node (o) at $t$. In particular, we call $x_{N}^{t}, x_{N}^{t-1}$, and $x_{0}^{t-1}$ the external nodes and the others the internal nodes. A circle node $x_{i}^{t}$ is connected with circle nodes $x_{j}^{t-1}\left(j \in \boldsymbol{I}_{i}\right)$ via a square node, which is associated with the function $f_{i}\left(\boldsymbol{x}_{\boldsymbol{I}_{i}}^{t-1}\right)$. If the function is a single-variable function (i.e., $\left|\boldsymbol{I}_{i}\right|=1$ ), then it is represented by an open square ( $\square$ ). Otherwise (i.e., $\left|\boldsymbol{I}_{i}\right|>1$ ), it is represented by a filled square $(\boldsymbol{\square})$. We associate the factor $\phi_{i}^{t} D\left[f_{i}\left(\boldsymbol{x}_{\boldsymbol{I}_{i}}^{t-1}\right), x_{i}^{t}\right]$ with a subnetwork called a bond that consists of a square, edges incident to the square, and circles neighboring the square. Examples of bonds are represented by bold and dotted lines in Fig. 2(a). Finally, we define the union of bonds (that is not empty) in the temporal graph as a pathway, $\alpha$, which plays a crucial role in TE [Figs. 2(a)-2(c)]. (As shown later, three of the pathways drawn in Fig. 2 are sufficient to identify the differences of TE between the coherent and incoherent motifs.)

If we are given an initial distribution $P\left(x^{t-q}\right)$ of the whole system at a distant past time $t-q$, we can write down the joint distribution $P\left(x_{N}^{t}, x_{N}^{t-1}, x_{0}^{t-1}\right)$ by using $P\left(\boldsymbol{x}^{t}\right)=$ $\sum_{x^{t-1}} P\left(\boldsymbol{x}^{t} \mid \boldsymbol{x}^{t-1}\right) P\left(\boldsymbol{x}^{t-1}\right)$ and Eq. (3) repeatedly for past times. Decomposing $P\left(x^{t-q}\right)$ as $P\left(x^{t-q}\right)=P\left(x^{t-q}\right)^{(\phi=0)}+$ $\Delta P\left(x^{t-q}\right)$, where $P\left(x^{t-q}\right)^{(\phi=0)}$ is the $\phi$-independent part, i.e., the uniform distribution when $\phi_{i}=0$ for all $i$, and $\Delta P\left(x^{t-q}\right)$ represents the remaining unknown part, we obtain

$$
\begin{aligned}
P\left(x_{N}^{t}, x_{N}^{t-1}, x_{0}^{t-1}\right)= & \left(\frac{1}{2}\right)^{1+(q-1)(N+1)} \sum_{x_{1}^{t-1}, \cdots, x_{N-1}^{t-1}} \sum_{\boldsymbol{x}^{t-2}} \cdots \sum_{\boldsymbol{x}^{t-q}}\left(1+\phi_{N}^{t} D\left[f_{N}\left(\boldsymbol{x}_{\boldsymbol{I}_{N}}^{t-1}\right), x_{N}^{t}\right]\right) \\
& \times \prod_{t^{\prime}=2}^{q} \prod_{i=0}^{N}\left(1+\phi_{i}^{t-t^{\prime}+1} D\left[f_{i}\left(\boldsymbol{x}_{\boldsymbol{I}_{i}}^{t-t^{\prime}}\right), x_{i}^{t-t^{\prime}+1}\right]\right)\left(P\left(\boldsymbol{x}^{t-q}\right)^{(\phi=0)}+\Delta P\left(\boldsymbol{x}^{t-q}\right)\right) .
\end{aligned}
$$

See the Supplemental Material (SM) for the derivation [49]. The expansion of the product of all $\left(1+\phi_{i}^{t} D\right)$ and $\left(P\left(x^{t-q}\right)^{(\phi=0)}+\Delta P\left(x^{t-q}\right)\right)$ in Eq. (4) tells us that the joint distribution consists of a constant term independent of any $\phi_{i}^{t} D$ and terms proportional to the product of one or multiple $\phi_{i}^{t} D$. With each of the latter set of terms naturally being mapped to a particular pathway, the joint distribution can be expressed as the sum over pathways:

$$
P\left(x_{N}^{t}, x_{N}^{t-1}, x_{0}^{t-1}\right)=\frac{1}{2^{3}}\left(1+\sum_{\alpha} \mathcal{P}_{\alpha}+\mathcal{O}\left(\phi^{q}\right)\right),
$$

where $\mathcal{P}_{\alpha}$ is the pathway weight associated with pathway $\alpha$, and is given by

$$
\begin{aligned}
\mathcal{P}_{\alpha} & =\frac{1}{2^{M_{\alpha}}} \sum_{\boldsymbol{x}_{\alpha}} \prod_{\text {bond } \in \alpha} \phi_{i}^{t} D\left[f_{i}\left(\boldsymbol{x}_{\boldsymbol{I}_{i}}^{t-1}\right), x_{i}^{t}\right] \\
& =\frac{1}{2^{M_{\alpha}}} \sum_{\boldsymbol{x}_{\alpha}} \prod_{\text {bond } \in \alpha} \phi_{i}^{t} \sigma\left(f_{i}\left(\boldsymbol{x}_{\boldsymbol{I}_{i}}^{t-1}\right)\right) \sigma\left(x_{i}^{t}\right) .
\end{aligned}
$$

Here, we have defined $\sigma(x) \equiv D[x, 1]$ and used $D[x, y]=$ $\sigma(x) \sigma(y)$. $\prod_{\text {bond } \in \alpha}$ represents the product of all bond factors $\phi D^{\prime} s$ in pathway $\alpha$ [Fig. 2(d)]. $\boldsymbol{x}_{\alpha}$ denotes a set of internal nodes $x_{i}^{t}$ within pathway $\alpha$, and $M_{\alpha}$ denotes the number of the internal nodes. In what follows, we abuse notation slightly and use $\mathcal{P}_{\alpha}$ both for a pathway and for its weight. The pathway weight in Eq. (6) is easily calculated because the summation $\sum_{\boldsymbol{x}_{\alpha}}$ can be separately carried out for each time. A technical remark is that, when deriving Eq. (5), the initial distribution $P\left(x^{t-q}\right)$ need not be known because its details are incorporated into $\mathcal{O}\left(\phi^{q}\right)$ (see SM [49]).

One may suspect that an enormous number of pathways would contribute to Eq. (5). However, because of the property $\sum_{x} \sigma(x)=0$, most of the possible pathways are not existent, namely $\mathcal{P}_{\alpha}=0$. It can be shown that an existent pathway must satisfy two structural conditions:

(C1): In the pathway, circle nodes that do not have nodes at their right sides must be one of the external nodes.

(C2): For every internal node in the pathway, if all squares that are directly connected with the node and located within 
the pathway are open $(\square)$, then the number of the open squares must be even.

For example, in Figs. 2(a)-2(c), pathways drawn by bold lines are existent, while those drawn by dotted lines are not.

Condition (C1) implies that, starting from any internal node in a pathway, one can reach one of the external nodes $x_{N}^{t}, x_{N}^{t-1}, x_{0}^{t-1}$ by moving forward in time. In other words, internal nodes can influence the external nodes only by causal chains of interactions. Condition (C2) comes from a property of single-variable Boolean functions and the mathematical identity $\sum_{x} \sigma(x)=0$. See the SM [49] for the derivations of these two conditions on pathway weights.

By substituting Eq. (5) into the definition of the entropy, and using $\ln (1+X)=\sum_{k=1}^{\infty} \frac{(-1)^{k+1}}{k} X^{k}(-1<X \leqslant 1)$, $H\left[x_{N}^{t}, x_{N}^{t-1}, x_{0}^{t-1}\right]$ can be expanded in terms of $\mathcal{P}_{\alpha}$. Since marginalization of Eq. (5) provides $P\left(x_{N}^{t-1}, x_{0}^{t-1}\right), P\left(x_{N}^{t}, x_{N}^{t-1}\right)$, and $P\left(x_{N}^{t-1}\right), H\left[x_{N}^{t-1}, x_{0}^{t-1}\right], H\left[x_{N}^{t}, x_{N}^{t-1}\right]$, and $H\left[x_{N}^{t-1}\right]$ can also be expanded in the same way. Combining the four kinds of $H$, we finally arrive at

$$
T_{0 \rightarrow N}=\sum_{k=2}^{\infty} \frac{(-1)^{k}}{k(k-1)} \sum_{\alpha_{1}} \cdots \sum_{\alpha_{k}} \mathcal{P}_{\alpha_{1}} \mathcal{P}_{\alpha_{2}} \cdots \mathcal{P}_{\alpha_{k}},
$$

where a pathway product $\mathcal{P}_{\alpha_{1}} \mathcal{P}_{\alpha_{2}} \ldots \mathcal{P}_{\alpha_{k}}$ must satisfy the following conditions (see SM [49]):

(C3): Among $k$ pathways $\left\{\mathcal{P}_{\alpha_{1}}, \ldots, \mathcal{P}_{\alpha_{k}}\right\}$, the external nodes, $x_{N}^{t}, x_{N}^{t-1}$, and $x_{0}^{t-1}$ appear $2(m+1), 2 m^{\prime}$, and $2\left(m^{\prime \prime}+1\right)$ times, respectively $\left(m, m^{\prime}, m^{\prime \prime} \in \mathbb{Z}_{\geqslant 0}\right)$.

(C4): The union of pathways $\mathcal{P}_{\alpha_{1}} \cup \cdots \cup \mathcal{P}_{\alpha_{k}}$ is connected in the temporal graph.

The combination of (C3) and (C4) indicates that the signal $x_{0}^{t-1}$ is connected to the output $x_{N}^{t}$ in a pathway product in Eq. (7), which can achieve information transfer. Thus, the graphical conditions $(\mathrm{C} 1)-(\mathrm{C} 4)$ for pathways and their products remarkably reduce the number of factors that must be taken into account.

\section{COHERENT AND INCOHERENT MOTIFS}

As a relevant application, we first consider the coherent and incoherent motifs [Fig. 1(a)] [42]. The circles and triangles in Fig. 3 [as well as Fig. 1(b)] show (nonperturbative) numerical results of TE for these motifs. They are computed according to its original definition Eq. (2), assuming that all $\phi$ 's are the same, i.e., $\phi_{i}=\phi$. We can see that the coherent motif has a larger TE than the incoherent motif for any $\phi \in(0,1)$. Note that the TE becomes 0 in two extremes: as $\phi \rightarrow 0$, the information is not transmitted since dynamics becomes completely random, and as $\phi \rightarrow 1$, the ambiguity about the future disappears since the dynamics becomes deterministic.

Now we apply our method to the two motifs to explain the origin of their difference in TE. First, we prepare the temporal graph associated with their regulatory network topology, and find existent pathways with up to a certain number of squares or $\phi$. Some pathways with up to $\phi^{5}$ are shown in Figs. 2(a)2(c). $\mathcal{P}_{3}$ and $\mathcal{P}_{4}$ correspond to the shortest route, $0 \rightarrow 1 \rightarrow N$, and the second shortest one, $0 \rightarrow 1 \rightarrow 2 \rightarrow N$, respectively, in the regulatory network in Fig. 1(a).

Then, we construct pathway products satisfying both (C3) and (C4). For example, $\mathcal{P}_{1} \mathcal{P}_{2}$ is not connected and does not

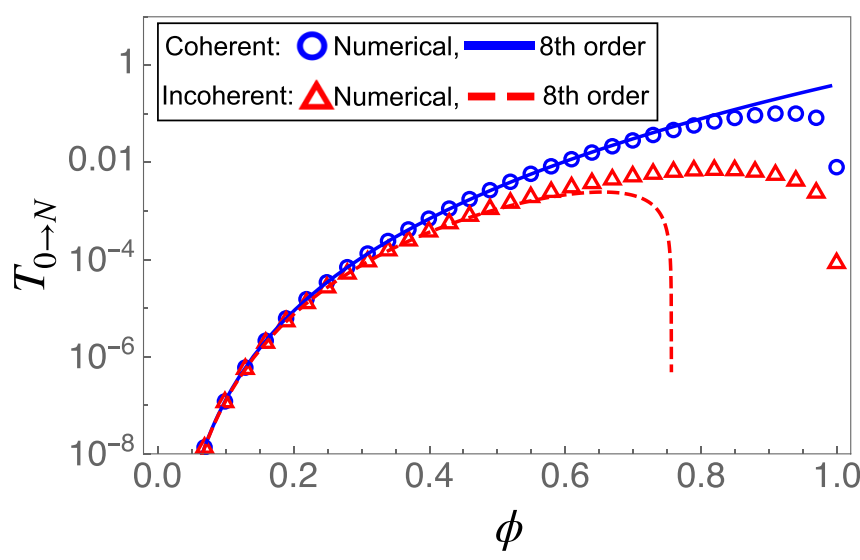

FIG. 3. The eighth-order perturbation results and the numerical nonperturbative ones for coherent (solid line, circle) and incoherent (dashed line, square) motifs.

satisfy (C4). $\mathcal{P}_{1}^{2}, \mathcal{P}_{1}^{3}$, and $\mathcal{P}_{1} \mathcal{P}_{3}$ are connected but do not satisfy (C3). The graphical conditions ensure that the first nonzero contribution always arises from the shortest route. In this case, it is given by $\mathcal{P}_{3}^{2}$, which has $\phi^{6}$. Physically, this represents the correlation between the signal $x_{0}^{t-1}$ and the output $x_{N}^{t}$ created by the past signal $x_{0}^{t-2}$ [see $\mathcal{P}_{3}$ in Fig. 2(b)]. The second leading terms are $\mathcal{P}_{1}^{2} \mathcal{P}_{3}^{2}$ and $\mathcal{P}_{3} \mathcal{P}_{4}$, which have $\phi^{8}$. Thus, up to $\phi^{8}$, the graphical expansion of TE is determined only by $\mathcal{P}_{1}, \mathcal{P}_{3}$, and $\mathcal{P}_{4}$, as written in Fig. 1(c), where binomial coefficients account for permutations of the pathways.

Pathway weights are computed from Eq. (6); for instance, $\quad \mathcal{P}_{3}=\frac{1}{2} \phi_{0} \phi_{1} \phi_{N} \sigma\left(x_{0}^{t-1}\right) \sigma\left(x_{N}^{t}\right) \quad$ and $\quad \mathcal{P}_{4}=$ $\pm \frac{1}{2} \phi_{0}^{2} \phi_{1} \phi_{2} \phi_{N} \sigma\left(x_{0}^{t-1}\right) \sigma\left(x_{N}^{t}\right)$, where the $+/-$ correspond to the coherent and incoherent motifs, respectively (see SM [49]). Finally, we obtain

$$
T_{0 \rightarrow N} \approx \frac{1}{8} \phi_{0}^{2} \phi_{1}^{2} \phi_{N}^{2}+\frac{1}{32} \phi_{0}^{2} \phi_{1}^{2} \phi_{N}^{4} \pm \frac{1}{4} \phi_{0}^{3} \phi_{1}^{2} \phi_{2} \phi_{N}^{2} .
$$

The difference in TE between the two motifs arises from the third term, $\mathcal{P}_{3} \mathcal{P}_{4}$, which is positive (negative) in the coherent (incoherent) motif [see also Fig. 1(c)]. This can be interpreted to mean that cooperative crosstalk between the two routes $0 \rightarrow 1 \rightarrow N$ and $0 \rightarrow 1 \rightarrow 2 \rightarrow N$ increases TE in the coherent motif, while disruptive crosstalk decreases it in the incoherent motif. In Fig. 3, the perturbative result, Eq. (8), is represented by the solid and dashed lines for the coherent and incoherent motifs, respectively. We can see that our method gives accurate results even for a large value of $\phi$ (i.e., $\phi \lesssim 0.7$ ), where the difference between the two motifs is quantitatively captured by the crosstalk term.

\section{OPTIMAL NETWORK ARCHITECTURE}

What features of the network dynamics determine the accuracy of information transfer? To answer this question, we focus on the leading contribution to the TE, which is $\mathcal{P}_{3}^{2}$ for the above examples. Suppose that there is a unique shortest route with length $L$; we denote it as $s(0)(=0) \rightarrow s(1) \rightarrow$ $\cdots \rightarrow s(L)(=N)$, where the indices $s(i)$ label the vertices along the route $(0 \rightarrow 1 \rightarrow N$ for the coherent or incoherent motif). By evaluating its pathway weight $\mathcal{P}_{\text {sh }}$ (schematically 


$$
\begin{array}{lllll}
t-L & \cdots & t-2 & t-1 \quad t
\end{array}
$$

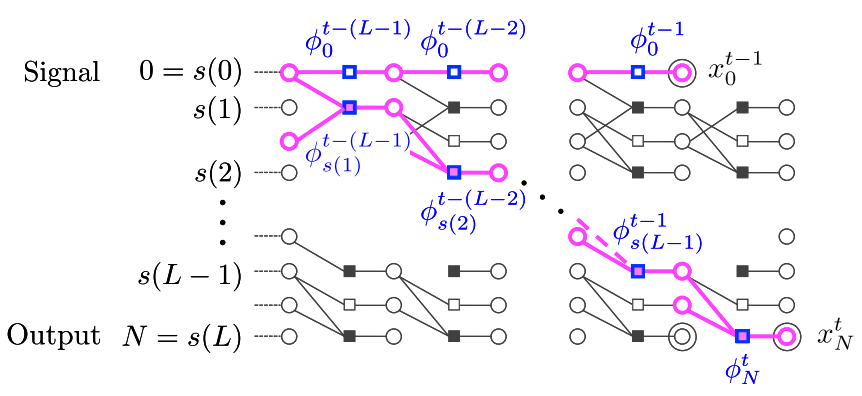

FIG. 4. Schematic of the shortest pathway $\mathcal{P}_{\text {sh }}$, which is associated with the shortest route, denoted by $s(0)(=0) \rightarrow s(1) \rightarrow \cdots \rightarrow$ $s(L)(=N)$ in the original regulatory network.

illustrated in Fig. 4) and substituting this in Eq. (7) $(k=2)$, the leading TE is given by

$$
T_{0 \rightarrow N}^{\text {leading }}=\frac{1}{2}\left(\mathcal{P}_{\mathrm{sh}}\right)^{2}=\frac{1}{2} \phi^{4 L-2} \prod_{i=1}^{L} C_{s(i)} .
$$

Here, $C_{s(i)}$ is the code correlation defined as

$$
C_{s(i)} \equiv \frac{1}{2^{2\left|\boldsymbol{I}_{s(i)}\right|}}\left(\sum_{\boldsymbol{x}_{\boldsymbol{I}_{(i)}}} \sigma\left(x_{s(i-1)}\right) \sigma\left(f_{s(i)}\left(\boldsymbol{x}_{\boldsymbol{I}_{s(i)}}\right)\right)\right)^{2} .
$$

See SM for the derivation [49]. Since $x_{s(i-1)}$ is an element of the inputs $\boldsymbol{x}_{\boldsymbol{I}_{s(i)}}$ of the function $f_{s(i)}, C_{s(i)}$ measures the correlation between the input $x_{s(i-1)}$ and the output $f_{s(i)} . C_{s(i)}$ takes a value in $[0,1]$. Under the condition of input-variables dependency that we mentioned earlier, $C_{s(i)}=1$ is possible only when $f_{s(i)}$ is a single-variable function.

An immediate consequence of the product expression in Eq. (9) is that we can examine the contribution to TE from each vertex one by one along the shortest route. We can show $C_{s(i)} \neq 0$ when $f_{s(i)}$ is monotonic, i.e., $f_{s(i)}$ satisfies, for any $j$, either $f_{s(i)}\left(x_{j}=1, \boldsymbol{x}_{\boldsymbol{I}_{s(i)} \backslash j}\right) \geqslant f_{s(i)}\left(x_{j}=0, \boldsymbol{x}_{\boldsymbol{I}_{s(i)} \backslash j}\right)$ for any state $\boldsymbol{x}_{\boldsymbol{I}_{s(i)} \backslash j}$ or $f_{s(i)}\left(x_{j}=1, \boldsymbol{x}_{\boldsymbol{I}_{s(i)} \backslash j}\right) \leqslant f_{s(i)}\left(x_{j}=0, \boldsymbol{x}_{\boldsymbol{I}_{s(i)} \backslash j}\right)$ for any state $\boldsymbol{x}_{\boldsymbol{I}_{s(i)} \backslash j}$. Furthermore, for maximization of $C_{s(i)}$, we can show that a multivariable function $f_{s(i)}$ is required to canalize in the input variable $x_{s(i-1)}$ along the shortest route (see SM [49] for the precise statement) [21,22], i.e., $f_{s(i)}\left(x_{s(i-1)}=x^{\prime}, \boldsymbol{x}_{\boldsymbol{I}_{s(i)} \backslash s(i-1)}\right)$ is uniquely determined independently of $\boldsymbol{x}_{\boldsymbol{I}_{s(i)} \backslash s(i-1)}$ for a certain $x^{\prime}$, whereas $f_{s(i)}\left(x_{s(i-1)}=\right.$ $\overline{x^{\prime}}, \boldsymbol{x}_{\boldsymbol{I}_{s(i)} \backslash s(i-1)}$ ) depends on $\boldsymbol{x}_{\boldsymbol{I}_{s(i)} \backslash s(i-1)}$ in general.

We demonstrate these expectations numerically for the network shown in Fig. 5(a) by considering each of the following three ensembles: (i) unbiased functions, i.e., those satisfying the dependency on the variables specified by the regulatory network, (ii) functions satisfying the dependency and monotonicity, and (iii) functions satisfying the dependency, monotonicity, and canalization along the shortest route. Figure 5(b) shows the histogram of the TE and the average. Consistent with our leading-order argument, the canalizing condition improves the TE drastically, while the monotonicity condition does so slightly. In the SM [49], we also evaluate the average and the maximum of the leading TE for this network (a)
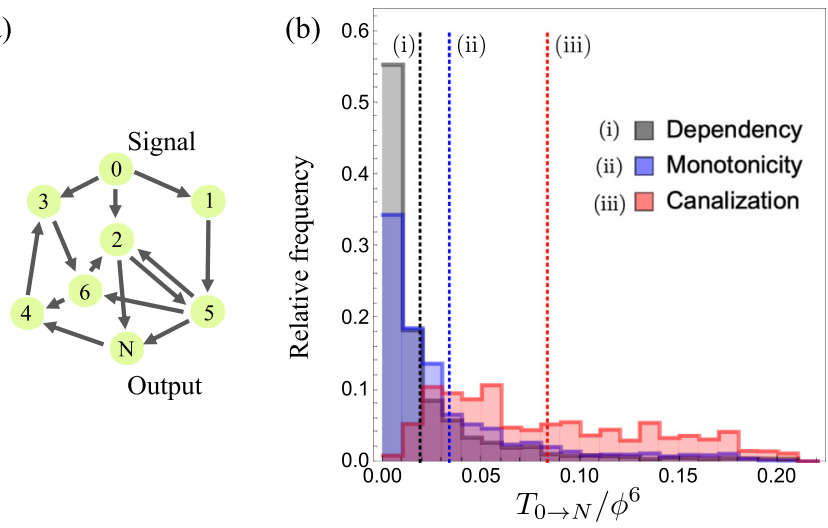

FIG. 5. (a) Random network with eight vertices. (b) Histograms and average (indicated by vertical lines) of TE in the random network, for the three ensembles of functions (i), (ii), and (iii), explained in the main text. $\phi=0.5$.

analytically and confirm that they qualitatively agree with the numerical results.

When there exist multiple $(S)$ shortest routes, Eq. (9) is modified to $T_{0 \rightarrow N}^{\text {leading }}=\frac{1}{2}\left(\sum_{i=1}^{S} \mathcal{P}_{\mathrm{sh}, i}\right)^{2}$. This indicates that, if all their weights $\mathcal{P}_{\mathrm{sh}, i}(i=1, \ldots, S)$ have the same sign, then the "redundancy" $S$ improves TE much more than linearly, $T_{0 \rightarrow N} \propto S^{2}$. On the other hand, if these weights cancel each other, the redundancy does not increase TE effectively. Thus, cooperative crosstalk between routes is essential, as in the coherent motif.

Our results provide several insights about information flow over real networks. First, a small diameter, one of the smallworld properties [13], provides an obvious advantage for information transfer, as the leading TE in Eq. (9) increases with a decrease in $L$. The small diameter is also important for the applicability of our formula because it allows us to compute the leading TE easily even for an extremely large network.

Another ubiquitous feature is a scale-free property [14], implying that signals pass through hubs. One may suspect that, unless functions are strongly biased (e.g., canalization), the signal information is likely to be lost at hubs, which receive many other inputs simultaneously. In fact, Eq. (10) suggests that, an unbiased function should receive a small number of inputs while its outdegree does not matter (see the SM [49]). Interestingly, in gene networks, indegrees are considerably smaller than outdegrees [50-53], which is indeed consistent with what our results suggest. Previous studies have reported similar findings. In Ref. [45], it has been numerically shown that the smallness of indegree on output vertex improves TE in random Boolean networks. In a model of policy diffusion [44], the outdegree of output vertex plays a marginal role in TE. Our results are consistent with these previous findings, although the details of these models and ours are different.

\section{DISCUSSION AND CONCLUSION}

Accurate information transfer is one of the most important functions for complex network systems. In this study, we have 
derived a general formula expressing TE in terms of graphical objects called pathways. We have obtained four conditions (C1)-(C4) that impose strong constraints on pathways and their products contributing to TE. The algorithm to compute TE is summarized as follows: For a given regulatory network, draw the corresponding temporal graph, and list up all pathways that satisfy conditions (C1) and (C2), up to a desired order in $\phi$. Then, using these pathways, construct pathway products that satisfy conditions (C3) and (C4), and sum them up according to Eq. (7). By automating these processes, information flow can be studied efficiently even in large complex networks.

Our formula provides a detailed understanding of how information flow is generated via network dynamics, as demonstrated in the coherent/incoherent example, in which we have identified the pathway interaction that gives rises to the difference in TE. Furthermore, we have obtained a concise expression of the leading TE as a product of correlations along the shortest route connecting the signal and output vertices. This result provides insights into graphical features essential for accurate information transfer. Our approach does not assume specific Boolean functions and is applicable to a wide class of Boolean networks.

Our diagrammatic method can be extended to other entropic quantities, such as mutual information, dissipation rate [54], and learning rate $[28,55]$, by employing different sets of external nodes and generalizing graphical conditions, which are the keys to reduce the number of pathways. Further applications of our technique will help to identify essential network structures and explore thermodynamical and informational aspects of real biological and technological systems.

While Boolean networks are used to study properties of large complex networks, there are several gaps between real systems and our framework presented in this paper. First, in our model, connection delays, i.e., time intervals for signal transmission between neighboring vertices, are identical to the unit time step. However, in real networks such as chemical reaction networks or gene regulatory networks, individual elements can have different connection delays. Formally, our framework can easily allow for arbitrarily long connection delays, by introducing bonds connecting nodes that are separated by two or more time steps in temporal graphs. Investigation of how heterogeneous connection delays, combined with network topology, affect information transfer in complex networks represents an interesting direction for further research.

Second, Eq. (1) implies an unbiased signal $x_{0}$, namely, $P\left(x_{0}^{t}=0\right)=P\left(x_{0}^{t}=1\right)=\frac{1}{2}$. However, a bias can be easily introduced by replacing $\phi_{0}^{t}$ with $\phi_{0}^{t}\left(x_{0}^{t-1}\right)$, i.e., by making $\phi_{0}^{t}$ dependent on the state $x_{0}^{t-1}$. In the presence of a bias, our graphical conditions must be modified because the bond associated with the signal, $\phi_{0}\left(x_{0}^{t-1}\right) D\left[x_{0}^{t-1}, x_{0}^{t}\right]$, is no longer eliminated by the summation over $x_{0}^{t-1}$. Another extension of our framework is the introduction of multiple signal variables with different temporal statistics. It would be interesting to elucidate how multiple signals improve transfer entropy.

There is another gap between our model and real networks. Nodes are modeled by Boolean variables in this study, but they may be better approximated in terms of nonBoolean state variables, such as multiple-state variables and continuum-state variables. For non-Boolean variables, the identity $\sum_{x} \sigma(x)=0$, which is critical for the reduction of pathways, does not hold. Therefore, a different mathematical technique must be developed. We leave this for future work.

\section{ACKNOWLEDGMENTS}

This work was supported by JSPS KAKENHI Grant No. JP19K03663 and the RIKEN iTHEMS Program.
[1] S. A. Kauffman, Metabolic stability and epigenesis in randomly constructed genetic nets, J. Theor. Biol. 22, 437 (1969).

[2] R. Albert and H. G. Othmer, The topology of the regulatory interactions predicts the expression pattern of the segment polarity genes in Drosophila melanogaster, J. Theor. Biol. 223, 1 (2003).

[3] F. Li, T. Long, Y. Lu, Q. Ouyang, and C. Tang, The yeast cellcycle network is robustly designed, Proc. Natl. Acad. Sci. USA 101, 4781 (2004).

[4] T. Gross and B. Blasius, Adaptive coevolutionary networks: A review, J. R. Soc. Interface 5, 259 (2008).

[5] I. Albert, J. Thakar, S. Li, R. Zhang, and R. Albert, Boolean network simulations for life scientists, Source Code Biol. Med. 3, 16 (2008).

[6] T. Helikar, J. Konvalina, J. Heidel, and J. A. Rogers, Emergent decision-making in biological signal transduction networks, Proc. Natl. Acad. Sci. USA 105, 1913 (2008).

[7] D. Sahoo, J. Seita, D. Bhattacharya, M. A. Inlay, I. L. Weissman, S. K. Plevritis, and D. L. Dill, MiDReG: A method of mining developmentally regulated genes using Boolean implications, Proc. Natl. Acad. Sci. USA 107, 5732 (2010).
[8] P. Moretti and M. A. Muñoz, Griffiths phases and the stretching of criticality in brain networks, Nat. Commun. 4, 2521 (2013).

[9] C. Seshadhri, A. M. Smith, Y. Vorobeychik, J. R. Mayo, and R. C. Armstrong, Characterizing short-term stability for Boolean networks over any distribution of transfer functions, Phys. Rev. E 94, 012301 (2016).

[10] F. Mori and A. Mochizuki, Expected Number of Fixed Points in Boolean Networks with Arbitrary Topology, Phys. Rev. Lett. 119, 028301 (2017).

[11] X. Gan and R. Albert, General method to find the attractors of discrete dynamic models of biological systems, Phys. Rev. E 97, 042308 (2018).

[12] B. C. Daniels, H. Kim, D. Moore, S. Zhou, H. B. Smith, B. Karas, S. A. Kauffman, and S. I. Walker, Criticality Distinguishes the Ensemble of Biological Regulatory Networks, Phys. Rev. Lett. 121, 138102 (2018).

[13] D. J. Watts and S. H. Strogatz, Collective dynamics of small-world networks, Nature (London) 393, 440 (1998).

[14] A.-L. Barabási and R. Albert, Emergence of scaling in random networks, Science 286, 509 (1999). 
[15] S. S. Shen-Orr, R. Milo, S. Mangan, and U. Alon, Network motifs in the transcriptional regulation network of Escherichia coli, Nat. Genet. 31, 64 (2002).

[16] R. Milo, S. Shen-Orr, S. Itzkovitz, N. Kashtan, D. Chklovskii, and U. Alon, Network motifs: Simple building blocks of complex networks, Science 298, 824 (2002).

[17] T. I. Lee, N. J. Rinaldi, F. Robert, D. T. Odom, Z. Bar-Joseph, G. K. Gerber, N. M. Hannett, C. T. Harbison, C. M. Thompson, I. Simon et al., Transcriptional regulatory networks in saccharomyces cerevisiae, Science 298, 799 (2002).

[18] E. Yeger-Lotem, S. Sattath, N. Kashtan, S. Itzkovitz, R. Milo, R. Y. Pinter, U. Alon, and H. Margalit, Network motifs in integrated cellular networks of transcription-regulation and protein-protein interaction, Proc. Natl. Acad. Sci. USA 101, 5934 (2004).

[19] B. Derrida and Y. Pomeau, Random networks of automata: A simple annealed approximation, Europhys. Lett. 1, 45 (1986).

[20] B. Derrida and D. Stauffer, Phase transitions in twodimensional Kauffman cellular automata, Europhys. Lett. 2, 739 (1986).

[21] A. A. Moreira and L. A. N. Amaral, Canalizing Kauffman Networks: Nonergodicity and Its Effect on Their Critical Behavior, Phys. Rev. Lett. 94, 218702 (2005).

[22] A. Szejka and B. Drossel, Evolution of canalizing Boolean networks, Eur. Phys. J. B 56, 373 (2007).

[23] T. Stephen and T. Yusun, Counting inequivalent monotone Boolean functions, Discrete Appl. Math. 167, 15 (2014).

[24] U.-U. Haus, S. Klamt, and T. Stephen, Computing knock-out strategies in metabolic networks, J. Comput. Biol. 15, 259 (2008).

[25] T. Schreiber, Measuring Information Transfer, Phys. Rev. Lett. 85, 461 (2000).

[26] S. Ito and T. Sagawa, Information Thermodynamics on Causal Networks, Phys. Rev. Lett. 111, 180603 (2013).

[27] D. Hartich, A. C. Barato, and U. Seifert, Stochastic thermodynamics of bipartite systems: Transfer entropy inequalities and a Maxwell's demon interpretation, J. Stat. Mech. (2014) P02016.

[28] J. M. Horowitz and M. Esposito, Thermodynamics with Continuous Information Flow, Phys. Rev. X 4, 031015 (2014).

[29] S. Ito and T. Sagawa, Maxwell's demon in biochemical signal transduction with feedback loop, Nat. Commun. 6, 7498 (2015).

[30] M. Bauer, J. W. Cox, M. H. Caveness, J. J. Downs, and N. F. Thornhill, Finding the direction of disturbance propagation in a chemical process using transfer entropy, IEEE Trans. Control Syst. Technol. 15, 12 (2006).

[31] D. Hartich, A. C. Barato, and U. Seifert, Sensory capacity: An information theoretical measure of the performance of a sensor, Phys. Rev. E 93, 022116 (2016).

[32] R. Vicente, M. Wibral, M. Lindner, and G. Pipa, Transfer entropy'a model-free measure of effectiveconnectivity for the neurosciences, J. Comput. Neurosci. 30, 45 (2011).

[33] K. Friston, R. Moran, and A. K. Seth, Analysing connectivity with Granger causality and dynamic causal modelling, Curr. Opin. Neurobiol. 23, 172 (2013).

[34] R. Marschinski and H. Kantz, Analysing the information flow between financial time series, Eur. Phys. J. B 30, 275 (2002).

[35] O. Kwon and J.-S. Yang, Information flow between stock indices, Europhys. Lett. 82, 68003 (2008).

[36] L. Sandoval, Structure of a Global network of financial companies based on transfer entropy, Entropy 16, 4443 (2014).
[37] O. Obst, J. Boedecker, and M. Asada, Improving recurrent neural network performance using transfer entropy, in International Conference on Neural Information Processing, ICONIP 2010 (Springer, Berlin, 2010), pp. 193-200.

[38] M. Staniek and K. Lehnertz, Symbolic Transfer Entropy, Phys. Rev. Lett. 100, 158101 (2008).

[39] M. Staniek and K. Lehnertz, Symbolic transfer entropy: inferring directionality in biosignals, Biomed. Tech. (Berlin) 54, 323 (2009).

[40] S. Ito, M. E. Hansen, R. Heiland, A. Lumsdaine, A. M. Litke, and J. M. Beggs, Extending transfer entropy improves identification of effective connectivity in a spiking cortical network model, PLoS One 6, e27431 (2011).

[41] S. Otsubo and T. Sagawa, Information-thermodynamic characterization of stochastic Boolean networks, arXiv:1803.04217.

[42] S. Mangan and U. Alon, Structure and function of the feedforward loop network motif, Proc. Natl. Acad. Sci. USA 100, 11980 (2003).

[43] M. Porfiri and M. R. Marín, Information flow in a model of policy diffusion: An analytical study, IEEE Trans. Netw. Sci. Eng. 5, 42 (2018).

[44] R. H. Goodman and M. Porfiri, Topological features determining the error in the inference of networks using transfer entropy, Math. Eng. 2, 34 (2020).

[45] L. Novelli, F. M. Atay, J. Jost, and J. T. Lizier, Deriving pairwise transfer entropy from network structure and motifs, Proc. R. Soc. A 476, 20190779 (2020).

[46] J. Runge, J. Heitzig, V. Petoukhov, and J. Kurths, Escaping the Curse of Dimensionality in Estimating Multivariate Transfer Entropy, Phys. Rev. Lett. 108, 258701 (2012).

[47] J. Runge, Quantifying information transfer and mediation along causal pathways in complex systems, Phys. Rev. E 92, 062829 (2015).

[48] P. Jiang and P. Kumar, Interactions of information transfer along separable causal paths, Phys. Rev. E 97, 042310 (2018).

[49] See Supplemental Material at http://link.aps.org/supplemental/ 10.1103/PhysRevResearch.2.043432 for the detailed derivations, the proofs, the numerical data, and the precise statements.

[50] T. Murali, S. Pacifico, J. Yu, S. Guest, G. G. Roberts, and R. L. Finley, DroID 2011: A comprehensive, integrated resource for protein, transcription factor, RNA and gene interactions for Drosophila, Nucleic Acids Res. 39, D736 (2011).

[51] J. R. Ecker, W. A. Bickmore, I. Barroso, J. K. Pritchard, Y. Gilad, and E. Segal, ENCODE explained, Nature (London) 489, 52 (2012).

[52] S. Gama-Castro, H. Salgado, A. Santos-Zavaleta, D. LedezmaTejeida, L. Muñiz-Rascado, J. S. García-Sotelo, K. AlquiciraHernández, I. Martínez-Flores, L. Pannier, J. A. CastroMondragón et al., RegulonDB version 9.0: High-level integration of gene regulation, coexpression, motif clustering and beyond, Nucleic Acids Res. 44, D133 (2016).

[53] N. Ichinose, T. Yada, and H. Wada, Asymmetry in indegree and outdegree distributions of gene regulatory networks arising from dynamical robustness, Phys. Rev. E 97, 062315 (2018).

[54] J. M. Horowitz, Multipartite information flow for multiple Maxwell demons, J. Stat. Mech. (2015) P03006.

[55] R. A. Brittain, N. S. Jones, and T. E. Ouldridge, What we learn from the learning rate, J. Stat. Mech. (2017) 063502. 